\title{
Das Margarin, seine Verdaulichkeit und sein Nährwerth im Vergleich zur reinen Natur- butter
}

\author{
von \\ Dr. Adolf Jolles in Wien. \\ (Vorgelegt in der Sitzung am 15. Februar 1894.)
}

Das Verdienst, aus thierischem Fett ein Surrogat der Naturbutter zuerst hergestellt zu haben, gebührt bekanntlich dem Franzosen Me'ge-Mouries, welcher von Napoleon III, aus socialökonomischen Gründen mit der Aufgabe betraut wurde, ein billiges Ersatzmittel der kostspieligen Naturbutter zu finden. Nachdem er durch Versuche seine Annahme bestätigt fand, dass das Milchfett seine Entstehung vornehmlich den im Thierkörper vorgebildet gewesenen Fetten verdanke, gelang es ihm thatsächlich, aus dem Thierfette durch künstliche Absonderung des festen Stearins, sowie eines Haupttheiles des Palmitins von dem flüssigbleibenden Oleïn in diesem ein Product zu erhalten, welches in seinen wesentlichsten Eigenschaften mit der Fettsubstanz der Kuhbutter übereinstimmte.

Gestützt auf diese Versuche gab er ein Verfahren zur fabriksmässigen Herstellung des Margarins, respective der Kunstbutter an, welches sich nach mannigfachen Verbesserungen auch in anderen Ländern, wie Österreich, Deutschland, Holland, Nord-Amerika Eingang verschaffte.

Bei der hohen Bedeutung, welche das Margarin gegenwärtig als Nahrungsmittel besitzt, erschien es von Interesse, die Frage der Verdaulichkeit und des relativen Nährwerthes des reinen Margarins einer möglichst exacten Untersuchung zu unterziehen. 
Leider finden wir über diese in hygienischer und ernährungsphysiologischer Beziehung so wichtigen Frage nur spärliche Angaben in der Literatur.

Eine Commission der medicinischen Akademie in Paris, welche sich über Auftrag des französischen Ministeriums des Inneren mit dieser Frage im Jahre 1880 beschäftigte, erklärte das Margarin als der Butter nicht gleichwerthig. Nach der Ansicht der Akademie werde das Margarin infolge seines grösseren Fettsäuregehaltes und infolge der Schwierigkeit der Umwandlung zu einer Emulsion im Darme nur unvollkommen resorbirt. Leider finden wir über die Producte, mit welchen die erwähnte Commission ihre Versuche durchgeführt, keine nähere Angabe und kennen die Arbeiten nicht, durch welche die Commission zu dem eben erwähnten Schlusse gelangte.

Wie wir schon bemerkten, spielt die Qualität des Materials eine wesentliche Rolle in der Margarinerzeugung, und es ist nicht ausgeschlossen, dass das Versuchsproduct der Commission noch verhältnissmässig reich an Stearin- und Palmitinsäure war, worauf möglicherweise das obige Gutachten zurückzuführen wäre.

Die angeblich schwierige Emulsion des Margarins im Darme konnten wir nicht constatiren, vielmehr haben wir bei einem zu Vergleichszwecken vorgenommenen künstlichen Versuche mit sogenanntem »Primissima-Margarin « und Naturbutter unter dem Mikroskope die gleiche Emulsionsbildung beobachtet.

In dem umfassenden technischen Gutachten, welches dem deutschen Reichstage zu dem Gesetzentwurf, betreffend den Verkehr mit Kunstbutter, vorgelegt wurde, wird ausgeführt, dass das Margarin, respective die Kunstbutter hinsichtlich ihres Nährwerthes hinter der reinen Naturbutter nicht wesentlich zurïckstehe, aber als etwas schwer verdaulich qualificirt werde. Diese gutachtliche Äusserung stützt sich auf keine in der Publication angeführten wissenschaftlichen Experimente. Sel1 ${ }^{1}$ gelangt zu dem Ergebnisse, $»$ dass die aus dem Fett gesunder Thiere dargestellte Kunstbutter, abge-

1 »Über Kunstbutter. - Ihre sanitäre Beurtheilung und die Mittel zu ihrer Unterscheidung von Mitchbutter \& (Arbeiten aus dem käiserl. Gosundheitsamte, 1. Band, $481-545$.) 
sehen von einer etwas geringeren Verdaulichkeit im Vergleich zul Milchbutter im Allgemeinen keine Veranlassung zu der Annahme gibt, dass sie auf die menschliche Gesundheit nachtheilig einwirken könne«.

Nach Prof. Uffelmann ${ }^{1}$ ist das Margarin, respective die Kunstbutter mbeinahe ebenso verdaulich $(96 \%)$ wie Naturbuttere. Leider erfahren wir auch hier nicht, wie Uffelmann zu dem Resultate $96 \%$ gelangt ist.

Prof. Flügge sagt in seinem ausgezeichneten Werke: "Grundriss der Hygiene (Verlag von Veit \& Comp.,1889, S.294) kurz und bündig: »In Bezug auf die Ausnützung und die Bedeutung als Fettnahrung ist die Kunstbutter der Naturbutter gleichwerthig «.

A. Mayer ${ }^{2}$ hat die Frage derVerdauung durch Ernährungsversuche zu lösen sich bemüht. Demzufolge wurden von zwei Personen beim täglichen Genusse von $62-70 \mathrm{~g}$. Butter die Milchbutter bis auf $2 \%$, die Kunstbutter bis auf $4 \%$, also nur wenig schlechter verdaut.

Nach Mayer ist in Übereinstimmung mit den vorstehenden Angaben »die Milchbutter leichter zu verdauen als Kunstbutter".

Auch von Mayer erfahren wir nicht, welche Qualität das zu seinen Versuchen verwendete Margarin besass, aber abgesehen hievon scheint es uns, dass eine so minimale Differenz wie $2 \%$, die ohne Bedenken als ein möglicher Versuchsfehler hingestellt werden könnte, kaum zu der obigen Behauptung berechtigt.

Herder empfiehlt in der $»$ Milch-Zeitung $(1887$, XVI, 360) die Verwendung des reinen, nicht zu Kunstbutterzwecken verarbeiteten Margarins zu Küchenzwecken auf Grund jahrelangen Gebrauches im eigenen Haushalte.

Wie also aus diesen, in der uns zur Verfügung gestandenen Literatur entnommenen Angaben hervorgeht, kann die Frage der Verdaulichkeit und des relativen Nährwerthes des reinen

1 *Handbuch der Hygiene * von Prof. Uffelmann. (Verlag von Urban und Schwarzenberg, 1890, S. 206.)

2 "Landwirthschaftliche Versuchsstation «, 29, S. 215. 
Margarins nach den Anforderungen der exakten Wissenschaft keineswegs als abgeschlossen betrachtet werden.

Diese Thatsache veranlasste uns, den Gegenstand einer neuerlichen möglichst exakten Untersuchung zu unterziehen, wobei wir, was ich hervorhebe, stets bedacht waren, nur reines Material zu verfüttern und unter möglichst gleichen Verhältnissen den Versuch durchzuführen.

Wir haben zur Feststellung des physiolog ischen Nährverthes von Margarin gegenüber Kuhbutter einen längeren Fütterungsversuch mit einem Hunde angestel1t.

Der Hund schien uns das geeignetste Versuchsthier, weil wir bei dem Hunde nicht mit den nervösen Zuständen zu rechnen brauchen, wie sie bei derartigen Versuchen an Menschen in mehr oder minder hohem Grade aufzutreten pflegen, weil ferner bei Fütterungsversuchen beim Menschen die Nahrung nicht in ebenso gleichmässiger Weise in Anwendung gebracht werden kann, wie bei Versuchen am Thiere, und endlich, weil bei dem Hunde der Ernährungs-, respective Verdauungsvorgang sich ähnlich wie bei dem Menschen abspielt.

Allerdings waren wir uns bewusst, dass bei einem so kleinen Versuchsthiere wie das unsere war, entsprechend den relativ geringeren Einnahmen und Ausgaben an Nährstoffen auch die etwaigen geringen Versuchsfehler bei den Resultaten schwer ins Gewicht fallen würden.

Wir waren daher um so mehr bemüht, die Fehlerquellen auf ein Minimum zu reduciren.

Das Thier war derart abgerichtet, dass es die Secrete und Excrete in eine bestimmte Schale entleerte und war während der Mahlzeit in einem Käfig eingesperrt, welcher durch seine Construction sämmtliche Secrete und Excrete aufzufangen gestattete.

Überdies war Vorsorge getroffen, dass das Thier sich während des Tages unter Aufsicht im Zimmer frei bewegen konnte, und wurden überhaupt alle jene Vorsichtsmassregeln eingehalten, welche bei derartigen Versuchen zu beachten sind.

Was nun die Behandlung des Hundes während des Versuches betrifft, so wurde derselbe täglich um $1 / 29$ Uhr Morgens gewogen, dann harnen gelassen und hierauf wieder gewogen. 
Dadurch wollten wir als eine weitere Controle erfahren, ob das Lebendgewicht vor und nach dem Harnen mit dem Harngewicht übereinstimmt. Selbstverständlich wurde an solchen Tagen, wo der Hund Koth abgab, auch der Koth berücksichtigt.

Die täglich aufgefangene Harnmenge wurde auf $17 \cdot 5^{\circ} \mathrm{C}$. abgekühlt, gewogen und unmittelbar darauf mittelst eines Urometers das specifische Gewicht festgestellt.

Ein Fünftel der täglichen Harnmenge wurde zur Bestimmung der Trockensubstanz auf dem Wasserbade zur Gewichtsconstanz eingedampft.

Für die Aschenbestimmung wurde der obige Abdampfrückstand in bei Harnen üblicher Weise verascht.

Die Stickstoffbestimmung wurde nach Kjeldahl vorgenommen, und zwar täglich zwei Stickstoffbestimmungen ausgeführt.

Trat überhaupt eine Differenz in diesen zwei Bestimmungen ein, dann wurde noch eine Controlbestimmung durchgeführt. Des weiteren wurde täglich ein Zehntel des Gesammtvolumens des Harnes zum Zwecke weiterer Untersuchungen, auf die wir noch im Laufe der Arbeit zurückkommen werden, eingedampft.

Was den Koth betrifft, so wurde derselbe nach dem Absetzen sorgfältig gesammelt und gewogen, dann bei circa $100^{\circ} \mathrm{C}$. bis zur Gewichtsconstanz getrocknet und abermals gewogen.

Der getrocknete Koth wurde gesammelt.

Der Gesammtkoth einer jeden Periode wurde fein vermahlen, gesiebt und gut durchgemischt. Leider waren stets im Kothe kleine Härchen enthalten, welche nach Möglichkeit entfernt wurden.

Zur N- und Fettbestimmung haben wir entsprechende sorgfältig entnommene Durchschnittsproben verwendet.

Die Fettbestimmungen geschahen in allen Fällen im Soxhlet'schen Apparate durch Extraction mit wasserfieiem Äther.

Über die weiteren Methoden, welche im Laufe der Arbeit in Anwendung kamen, berichten wir an den betreffenden Stellen. 
Anlangend die eigentliche Fütterung, haben wir zunächst die ganze Fütterungsdauer in vier Perioden eingetheilt, wobei in den Perioden I und III Naturbutter und in den Perioden II und IV Margarin zur Verfütterung gelangte.

Ferner haben wir in den Perioden I und II Fett und weniger Kohlehydrate, in den Perioden III und IV weniger Fett und mehr Kohlehydrate verfüttert, um zu ersehen, wie bei vermehrter oder verminderter Anwesenheit der fett- und eiweisssparenden Kohlehydrate sich die Ausnützung der beiden Fette. verhalte.

Wir wählten ferner auch längere Perioden von mindestens acht Tagen Dauer und verfolgten damit den Zweck, durch die Länge der Perioden die Fehlerquellen zu verringern, welche durch die verhältnissmässig geringe Einfuhr und also auch Ausfuhr von Nährstoffen hervorgerufen werden können.

Das dem Versuchsthiere gereichte Futter bestand aus Fett, Kohlehydraten, Eiweisstoffen und Salzen.

Das Fett war bei den Perioden I und IIJ reine Kuhbutter, bei den Perioden II und IV reines Margarin.

Als Kohlehydrate gelangten Weizenmehl 000 und Raffinadezucker in Verwendung, und als Eiweissstoffe wählten wir statt Fleischmehl Aleuron, ein durch Dr. Hundhausen in Hamm aus Weizenkleber hergestelltes eiweissreiches Präparat.

$\mathrm{Zu}$ der Verwendung von Aleuron statt Fleisch als Grundfutter veranlasste uns eine Arbeit von Dr. Kornauth, "Über Fütterungsversuche mit Aleuronat " ${ }^{l}$ in der Verfasser zu dem Ergebnisse gelangte, dass das Aleuron in hohem Grade verdaulich und total unveränderlich sei.

Auch Prof. Gruber schliesst aus seinen Versuchen mit Aleuronbrot, dass die Ausnützung des Aleurons als ganz vortrefflich bezeichnet werden muss.

Aus den erwähnten Rohmaterialien, über deren chemische Zusammensetzung wir noch im speciellen. Theile unserer Arbeit zurückkommen, haben wir kleine runde Cakes herstellen lassen, die sich durch angenehmen Geruch und Geschmack und was für uns besonders wichtig war - durch Gleichmässigkeit

1 Österreichisches landwirthschaftliches Centralblatt, 1892. 
und Unveränderlichkeit ausgezeichnet haben. Die in den Perioden I und III verfütterten Cakesmengen wurden unter Butterzusatz, die in den Perioden II und IV unter Margarinzusatz hergestellt. Ausserdem erhielt das Thier pro Tag genau bestimmte Mengen von reiner Naturbutter, respective reinem Margarin. Während der Fütterungsperiode erhielt der Hund täglich um 9 Uhr Morgens die abgewogene Menge der Cakes und des Fettes, sowie ausserdem pro Tag $300 \mathrm{~cm}^{3}$ Wasser.

Der Futterbehälter wurde täglich gereinigt.

Da bei unserem Fütterungsversuche das Fett eine so wichtige Rolle spielte, haben wir naturgemäss sehr hohe Fettmengen zu verfüttern gesucht und es dabei für zweckmässig gehalten, jeden Morgen die Fütterung durch Verabreichen der Fettmengen einzuleiten.

Nachdem der Hund in der Regel Morgens einen grösseren Appetit entwickelte, so hatte er auch das Fett gleich ganz oder zum grössten Theile gefressen und war dann die übrige Zeit auf die Cakes - die ihm in ganz kleinen Stücken verabreicht wurden - angewiesen. Um den Geschmack der Cakes in irgend einer Weise etwas zu ändern, wurden dann und wann die Cakes an einzelnen Stellen sehr fein mit Speck bestrichen, was genügte um die Esslust des Hundes anzuregen. Selbstverständlich konnte durch diesen Vorgang von einer Fetterhöhung nicht die Rede sein. Begreiflicherweise war die andauernde Verabreichung verhältnissmässig grosser Fettmengen dadurch unthunlich, dass der Hund an manchen Tagen die ihm dargereichte Nahrung nicht vollständig fressen wollte. Dadurch wurde es auch unmöglich, während der ersten und zweiten Fütterungsperiode den Hund mit absolut gleichen Mengen von Nährstoffen zu füttern.

Selbstverständlich sind wir aber vor jeder Fütterungsperiode vom Stickstoffgleichgewichte ausgegangen, in welchem dann der Hund bis zum Schlusse verblieb.

Die nachfolgenden Tabellen zeigen, dass die Gewichtsconstanz des Hundes und die minimale Menge von angesetztem $\mathrm{N}$ vollkommen im Einklange stehen. 


\section{Analysen der Rohmaterialien.}

Behufs Beschaffung von ganz reinen Rohmaterialien ersuchten wir die Wiener Molkerei um Überlassung von ganz reinen Butterproben, indem wir ausdrücklich hinzufügten, dass die Proben zu wissenschaftlichen Zwecken benöthigt werden. Die uns übermittelten Proben waren sogenannte »Theebutterproben v von sehr angenehmen Geruch und Geschmack und hatten folgende Zusammensetzung:

\begin{tabular}{|c|c|c|}
\hline & $\begin{array}{l}\text { Probe I } \\
\text { verfüttert in der } \\
\text { ersten Periode }\end{array}$ & $\begin{array}{l}\text { Probe il } \\
\text { verfüttert in de: } \\
\text { zwciten Perinde }\end{array}$ \\
\hline Wasser. & $10 \cdot 24 \%$ & $10 \cdot 33^{\circ} / 1$ \\
\hline Caseïn .... & $0 \cdot 63$ & $0 \cdot 60$ \\
\hline Milchzucker ............ & $0 \cdot 54$ & $0 \cdot 52$ \\
\hline Salze ............... & $0 \cdot 3 \pm$ & $0 \cdot 31$ \\
\hline Fett (Differenz)... & $88 \cdot 25$ & $88 \cdot 24$ \\
\hline Reichert-Meissl'sche Zahl. & $28 \cdot 6$ & $28 \cdot 2$ \\
\hline
\end{tabular}

Die Zusammensetzung der bei unseren Fütterungsversuchen in Verwendung gelangten Margarinproben, welche wir über Ersuchen von der »Wiener Margarin-Compagnie "zur Verfügung gestellt erhielten, war folgende:

$\begin{array}{cc}\text { Margarin, } & \text { Margarin, } \\ \text { verfüttert in der } & \text { verfüttert in der } \\ \text { Periode II } & \text { Periode IV }\end{array}$

Jodzahl ............... $47 \quad 47$

Schmelzpunkt der Fettsäuren... $42 \cdot 6^{\circ} \mathrm{C} . \quad 42 \cdot 6^{\circ} \mathrm{C}$.

Erstarrungspunkt der Fettsäuren. $39.5^{\circ} \mathrm{C} . \quad 39.5^{\circ} \mathrm{C}$.

Verseifungszahl .......... $197 \cdot 5 \quad 197 \cdot 5$

Ausser den erwähnten Fetten dienten als weitere Rohmaterialien Aleuron, Weizenmeh1 000, Rohrzucker (Raffinade) und Kochsalz. 
Margarin.

Analyse der Rohmaterialien: Aleuron und Weizenmehl 000.

Tabelle 1.

\begin{tabular}{|c|c|c|c|c|c|c|c|c|}
\hline & \multirow[b]{2}{*}{ 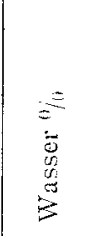 } & \multicolumn{7}{|c|}{ In der Trockensubstanz $0 / 1$} \\
\hline & & $\begin{array}{l}10 \\
01 \\
0 \\
7 \\
\stackrel{5}{7} \\
7\end{array}$ & 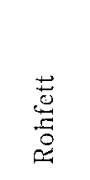 & 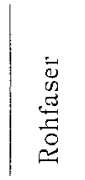 & 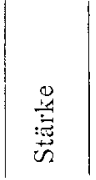 & 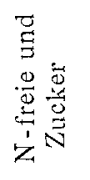 & $\begin{array}{l}\stackrel{0}{0} \\
\frac{0}{4}\end{array}$ & $N$ \\
\hline Weizenmeh1 000 & $12 \cdot 44$ & $10 \cdot 92$ & $1 \cdot 10$ & $0 \cdot 20$ & $85 \cdot 56$ & $1 \cdot 74$ & $0 \cdot 48$ & $1 \cdot 74$ \\
\hline Aleuronat .. & $5 \cdot 93$ & $86 \cdot 00$ & $1 \cdot 31$ & 0.06 & $6 \cdot 06$ & $5 \cdot 70$ & $0 \cdot 87$ & $13 \cdot 76$ \\
\hline
\end{tabular}

Aus den angegebenen Rohmaterialien wurden die Cakes I, II, III und IV für die vier Perioden hergestellt, und zwar vom Bäcker Herrn L. Schmidt in Wien, der die Cakes unter sorgfältigster Einhaltung aller ihm angegebenen Vorschriften in vorzüglichster Weise gebacken hatte.

Die für jede Periode bestimmten Cakes wurden nach sorgfältiger Entnahme einer Durchschnittsprobe analysirt und lieferte die Analyse folgendes Resultat:

\section{Analysen der Cakes für die Perioden.}

Tabelle 2.

\begin{tabular}{|c|c|c|c|c|c|c|c|c|}
\hline & \multicolumn{8}{|c|}{ In der Trockensubstanz } \\
\hline & Wasser & $\begin{array}{c}\text { Roh- } \\
\text { protein }\end{array}$ & Fett & Faser & $\left|\begin{array}{c}\text { N-freie } \\
\text { und } \\
\text { Zucker }\end{array}\right|$ & Stärke & Asche & $\begin{array}{l}\text { Stick- } \\
\text { stoff }\end{array}$ \\
\hline I. . & $4 \cdot 00$ & $34 \cdot 50$ & $19 \cdot 80$ & 0.06 & $42 \cdot 72$ & - & $2 \cdot 92$ & $5 \cdot 52$ \\
\hline II . . & $4 \cdot 80$ & $31 \cdot 50$ & $23 \cdot 10$ & $0 \cdot 05$ & $42 \cdot 33$ & - & $3 \cdot 02$ & $5 \cdot 04$ \\
\hline III. . & $7 \cdot 35$ & $21 \cdot 25$ & $12 \cdot 68$ & $0 \cdot 05$ & 64.02 & - & $2 \cdot 00$ & $3 \cdot 40$ \\
\hline IV...... & $6 \cdot 83$ & $21 \cdot 75$ & $14 \cdot 29$ & $0 \cdot 07$ & $61 \cdot 91$ & - & $1 \cdot 98$ & $3 \cdot 48$ \\
\hline
\end{tabular}

Die Mengen an Cakes und Fett, die der Hund pro Tag während jeder der vier Perioden verzehrte, stellen sich, wie folgt: 
Gesammtmenge der täglich verabreichten Nährstoffe.

Tabelle 3.

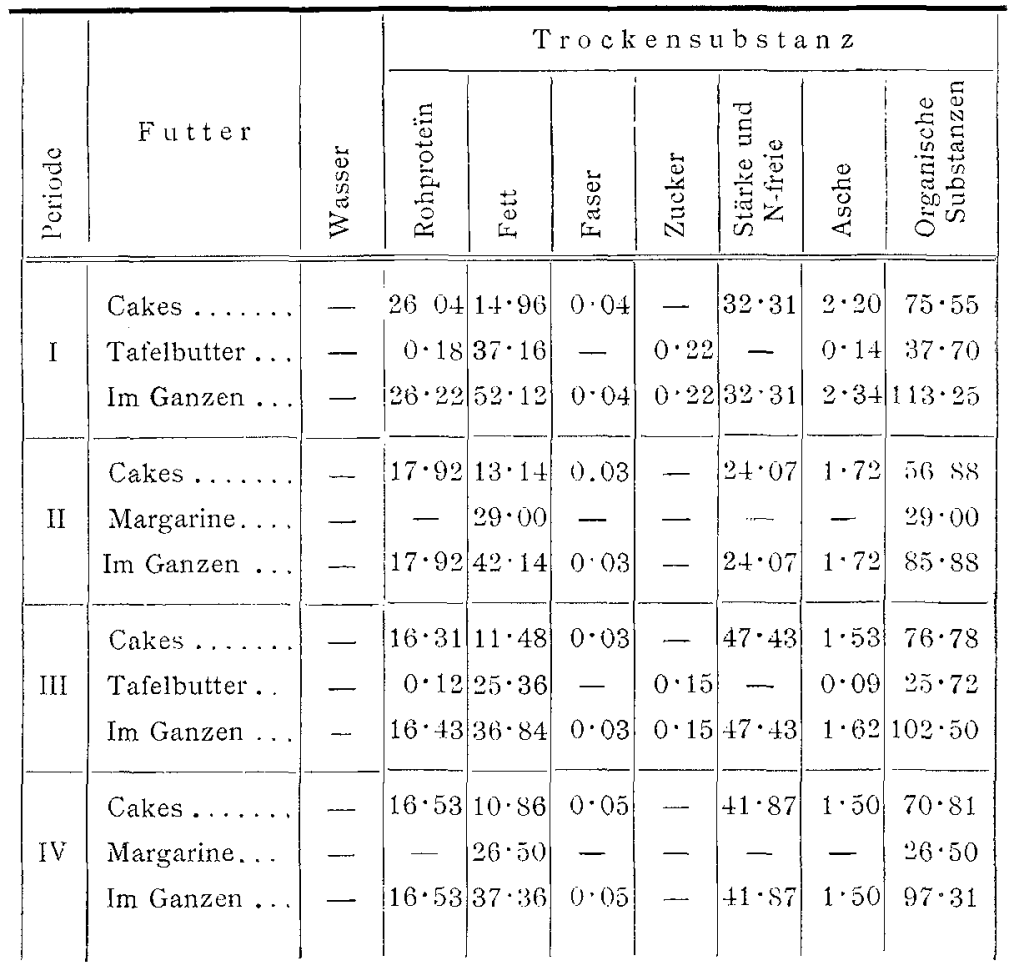

Aus dieser Tabelle geht zunächst hervor, dass in den Perioden III und IV der Hund fast gleiche Mengen an Nährstoffen verzehrte. Hingegen ist. in den Perioden I und II in Bezug auf die verabreichten Nährstoffe eine Differenz zu verzeichnen, die darauf zurückzuführen ist, dass der Hund die anfänglich verabreichte Futterration wegen des unverhältnissmässig hohen Fettgehaltes auf die Dauer nicht vertrug.

Selbstverständlich gingen wir bei allen Perioden, also auch bei der zweiten Periode, vom N-Gleichgewichte aus, und die nachfolgende Tabelle über die mittleren Zahlen für Lebendgewicht, Harn und Koth pro Tag zeigt, dass die minimalen Mengen von angesetztem Stickstoff mit der Gewichtsconstanz des Hundes in allen Perioden im vollsten Einklange stehen. 
Mittlere Zahlen für Lebendgewicht, Harn und Koth pro Tag.

Tabelle 4.

\begin{tabular}{|c|c|c|c|c|c|c|c|c|c|c|c|c|}
\hline \multirow{4}{*}{$\begin{array}{l}0 \\
\stackrel{0}{0} \\
\stackrel{0}{0} \\
\end{array}$} & \multirow{4}{*}{ 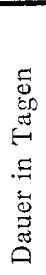 } & \multirow[b]{2}{*}{$\begin{array}{l}\frac{1}{0} \\
\stackrel{\bar{J}}{0} \\
\frac{0}{0} \\
-3\end{array}$} & \multirow[b]{2}{*}{ 䭴 } & \multirow{4}{*}{ 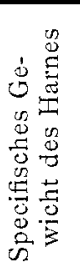 } & \multirow{2}{*}{ 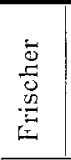 } & \multirow{2}{*}{ 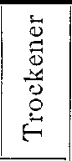 } & \multirow{3}{*}{ 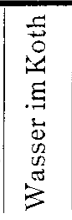 } & \multicolumn{5}{|c|}{ Vom Stickstoff sind } \\
\hline & & & & & & & & \multirow[t]{2}{*}{ 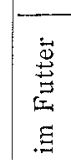 } & \multirow{2}{*}{$\begin{array}{c}\underset{E}{E} \\
\stackrel{\Xi}{\Xi} \\
\Xi\end{array}$} & \multirow{2}{*}{ 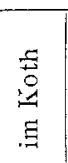 } & \multirow{2}{*}{$\begin{array}{l}\vec{N} \\
\text { U } \\
0 \\
0 \\
0 \\
\stackrel{0}{0} \\
\Xi\end{array}$} & \multirow{2}{*}{$\begin{array}{l} \\
0 \\
0 \\
80 \\
80 \\
80 \\
00 \\
0\end{array}$} \\
\hline & & \multicolumn{2}{|c|}{ Gewicht } & & $\mathrm{KC}$ & th & & & & & & \\
\hline & & \multicolumn{2}{|c|}{ Gramme } & & \multicolumn{8}{|c|}{ Gram $\mathrm{me}$} \\
\hline I & 10 & 5992 & $146 \cdot 5$ & $1 \cdot 033$ & $18 \cdot 0$ & $7 \cdot$ & 08 & $4 \cdot 21$ & $3 \cdot 88$ & $0 \cdot 27$ & 0.06 & - \\
\hline II & 8 & 5966 & $112 \cdot 5$ & $1 \cdot 035$ & 15 & 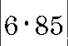 & $42 \cdot 7$ & $2 \cdot 86$ & $2 \cdot 56$ & $0 \cdot 29$ & 0.01 & - \\
\hline III & 8 & 5961 & $104 \cdot 0$ & $1 \cdot 035$ & & ( & $41 \cdot 5$ & $2 \cdot 64$ & $2 \cdot 11$ & $0 \cdot 46$ & 0.07 & - \\
\hline IV & 8 & 5930 & $99 \cdot 1$ & $1 \cdot 037$ & $13 \cdot 3$ & $5 \cdot 96$ & $49 \cdot 1$ & $2 \cdot 62$ & $2 \cdot 11$ & $0 \cdot 40$ & $0 \cdot 01$ & - \\
\hline
\end{tabular}

Die Tabellen erweisen ferner, dass bei jeder Periode von dem verabreichten Stickstoff nur ein sehr geringer Theil angresetzt wurde.

Diese Zahlen sind so minimal, dass wir sie keineswegs als Beweis für einen geringen Eiweissansatz verwerthen wollen, vielmehr sind sie für uns nur beweisend, dass im Anbeginn jeder Fütterungsperiode vom $\mathrm{N}$-Gleichgewichte ausgegangen worden war, und dass der Hund bis zum Schlusse einer jeden Periode auch im Gleichgewichte verblieben ist.

Von grosser Bedeutung war auch die weitere Frage, welche Mengen an Fett in Anbetracht der bedeutenden zur Verfütterung. gelangten Fettquantitäten unverdaut mit dem Kothe ausgeschieden wurden.

Zur Beantwortung dieser Frage haben wir die absolute Menge des mit dem Kothe ausgeschiedenen $\ddot{A}$ therextractes und dessen Antheil an der Trockensubstanz des Kothes festgestellt.

\begin{tabular}{|c|c|}
\hline Periode & Ätherextract \\
\hline \hline I & 0.795 \\
II & 0.86 \\
III & 1.05 \\
IV & 1.22 \\
\hline
\end{tabular}


Diese Zahlen lehren unter Hinweis auf Tabelle 3, dass in allen Perioden der bei weitem grösste Theil des verfütterten Fettes resorbirt und nur ein sehr geringer Theil mit dem Kothe ausgeschieden wurde, wozu auch noch kommt, dass ja von den Stoffwechselproducten ein Theil in Äther löslich ist. Auch geht aus den Thatsachen hervor, dass diesbezüglich zwischen den einzelnen Perioden nur unwesentliche Differenzen zu verzeichnen sind. (Wenn man überhaupt auf Grund der erhaltenen Daten von einer Differenz sprechen darf, so sei auf die geringe Differenz in Bezug auf den Ätherextract bei den Perioden III und IV gegenüber den Perioden I und II hingewiesen.)

Es liegt die Möglichkeit nahe, dass die in den Perioden III und IV erhöhte Verfütterung von Kohlehydraten, welchen bekanntlich eine ähnliche Rolle wie dem Fette bei der Ernährung zugeschrieben wird, zu der etwas geringeren Fettresorption in diesen Perioden beigetragen habe.

Das mit dem Kothe abgegangene Fett haben wir weiter untersucht, um festzustellen, ob dasselbe unverändertes, also vom Körper nicht resorbirtes Fett darstellt, oder ob dieses Fett aus Substanzen, welche durch im Körper hervorgerufene Zersetzungen entstanden sind, oder aus Gemischen beider besteht.

$\mathrm{Zu}$ diesem Zwecke haben wir das Ätherextract auf den Gehalt an freien Fettsäuren, Neutralfett und Cholesterin nach den Methoden, wie sie von Prof. Dr. Benedikt in seinem Werke: "Analyse der Fette und Wachsarten", II. Auflnge, angegeben ist, untersucht.

In 100 Theilen Ätherextract waren enthalten:

\begin{tabular}{|c|c|c|c|}
\hline Periode & $\begin{array}{c}\text { Freie } \\
\text { Fettsäuren }\end{array}$ & $\begin{array}{c}\text { Neutrales } \\
\text { Fett }\end{array}$ & Cholesterin \\
\hline \hline I & $56 \cdot 96 \% / 15$ & $37 \cdot 97 \% / \%$ & $5 \cdot 07 \% \%$ \\
II & $55 \cdot 81$ & $38 \cdot 37$ & $5 \cdot 92$ \\
III & $54 \cdot 28$ & $49 \cdot 00$ & $5 \cdot 72$ \\
II & 5491 & $41 \cdot 80$ & $8 \cdot 20$ \\
\hline
\end{tabular}


und berechnet auf:

\section{Gramm pro Tag.}

\begin{tabular}{|c|c|c|c|}
\hline Periode & $\begin{array}{c}\text { Freie } \\
\text { Fettsäuren }\end{array}$ & $\begin{array}{c}\text { Neutrales } \\
\text { Fett }\end{array}$ & Cholesterin \\
\hline \hline \multirow{2}{*}{ I } & 0.45 & 0.30 & 0.04 \\
II & 5.48 & 0.33 & 0.03 \\
III & 0.57 & 0.42 & 0.06 \\
IV & 0.67 & 0.51 & 0.04 \\
\hline
\end{tabular}

Aus diesen Tabellen geht hervor, dass die geringen Fettmengen, welche mit dem Kothe abgingen, zum grössten Theil in einer durch den Verdauungsprocess entstandenen Form zur Ausscheidung gelangt sind (freie Fettsäuren, Cholesterin) und mit ganz geringen Theilen unverändert den Verdauungstract passirte (Neutralfett).

Von Belang ist, dass der Gehalt an Neutralfett, ebenso wie der Gehalt an freien Fettsäuren und Cholesterin in sämmtlichen Perioden nahezu constant blieb.

Was nun die stickstoffhaltigen Bestandtheile des Kothes betrifft, so haben wir dieselben zunächst nach der Methode von Stutzer in Proteïn- und Nichtproteïnverbindungen zerlegt.

Wir erhielten folgende Resultate:

Pro $100 \mathrm{~g}$ Stickstoff sind im Kothe enthalten in Formen von:

\begin{tabular}{|c|c|c|}
\hline Periode & $\begin{array}{c}\text { Nichteiweiss- } \\
\text { stickstoff }\end{array}$ & $\begin{array}{c}\text { Eiweiss- } \\
\text { stickstoff }\end{array}$ \\
\hline \hline I & $23 \cdot 33$ & $76 \cdot 67$ \\
III & $28 \cdot 96$ & 71.04 \\
III & 21.95 & 78.05 \\
IV & 29.62 & $70 \cdot 35$
\end{tabular}


Im Koth pro Tag in Grammen.

\begin{tabular}{|c|c|c|}
\hline Periode & $\begin{array}{c}\text { Nichteiweiss- } \\
\text { stickstoff }\end{array}$ & $\begin{array}{c}\text { Eiweiss- } \\
\text { stickstoff }\end{array}$ \\
\hline I & 0.063 & 0.207 \\
II & 0.084 & 0.206 \\
III & 0.101 & 0.359 \\
IV & 0.117 & 0.278 \\
\hline
\end{tabular}

Aus diesen Tabellen geht die interessante Thatsache hervor, dass in den Perioden II und IV, also in den Margarinperioden die Menge des im Kothe wieder als Eiweiss auffindbaren Stickstoffes geringer war, als in den Perioden I und III, was zu Gunsten einer besseren Eiweissausnützung nach dem Genusse des Margarins gedeutet werden könnte. Diese Differenz ist aber unserer Ansicht nach eher auf den Umstand zurückzuführen, dass die geringen Caseïnmengen der Naturbutter infolge der unverhältnissmässig hohen Fettfütterungen nicht jene normale Verdauung gefunden haben, wie es unter normalen Verhältnissen der Fall ist, und dass daher diese Stoffe durch den Magen und Darm unverdaut mit dem Kothe abgingen.

Anderseits ist der Nicht-Eiweissstickstoff in den Perioden I und III etwas niedriger als in den Butterperioden II und IV, was nur darauf zurückgeführt werden kann, dass zur Zeit der Margarinfütterung eine etwas grössere Eiweisszersetzung oder eine etwas grössere Abspaltung von Amidostickstoff vor sich ging.

Beide übrigens unwesentliche Differenzen kommen, unserer Ansicht nach, bei der von uns behandelten Frage der Verdaulichkeit des Margarins im Vergleiche zur Naturbutter nicht weiter in Betracht.

Um $z u$ ersehen, ob der mit dem Kothe abgegangene Eiweissstickstoff im Magensafte und in der Pancreaslösung löslich (verdaulich) sei, haben wir den Eiweissstickstoff der 
vier Perioden nach Stutzer in verdauliche und unverdauliche Stickstoffverbindungen geschieden.

Von dem Eiweissstickstoff erwiesen sich in den vier Perioden bei der künstlichen Verdauung:

\begin{tabular}{|c|c|c|c|c|c|c|c|c|}
\hline & \multicolumn{7}{|c|}{ P e r i o d e } \\
\cline { 2 - 7 } & I & II & III & IV & I & II & III & IV \\
\hline & & in Grammen & & in Percenten \\
\hline Verdaulich.. & $0 \cdot 083$ & $0 \cdot 096$ & $0 \cdot 132$ & $0 \cdot 145$ & $30 \cdot 93$ & $33 \cdot 25$ & $28 \cdot 81$ & $36 \cdot 78$ \\
\hline Unverdaulich & $0 \cdot 187$ & $0 \cdot 194$ & $0 \cdot 328$ & $0 \cdot 250$ & $69 \cdot 07$ & $66 \cdot 75$ & $71 \cdot 19$ & $63 \cdot 22$ \\
\hline
\end{tabular}

Wenngleich aus dieser Tabelle einerseits resultirt, dass in allen Perioden der unverdauliche (ausgeschiedene) Eiweissstickstoff den verdaulichen bei weitem übertrifft, so ersieht man doch anderseits, dass in allen vier Perioden noch ein bedeutender Antheil von im Magensaft- und in der Pancreaslösung verdaulichen Eiweisssticksoff excernirt wurde. Meiss1, ebenso Kornauth und Arche haben in ihren Arbeiten »Über: den Stoffwechsel des Schweines ebenfalls von dem Eiweissstickstoff des Kothes eine verhältnissmässig hohe Verdaulichkeitsziffer gefunden, und Meissl gab einer auch bei unseren Versuchen naheliegenden Vermuthung Raum, dass nur infolge des raschen Durchgehens der Nahrung durch den Magen und Darm unverdautes Eiweiss mit dem Kothe abginge.

Berechnen wir nunmehr auf Grund der angeführten Daten die Verdaulichkeitscoëfficienten, respective die Ausnützung des Futters in den einzelnen Perioden, und zwar einfach in der Weise, dass zwischen Futter und Koth die Differenz gebildet wird, so resultirt folgende Tabelle:

$1 \gg$ Die landwirthschaftlichen Versuchs-Stationen«, Bd. XL, 1892. 
Tabelle über die Ausnützung des Futters.

\begin{tabular}{|c|c|c|c|c|c|c|c|c|}
\hline \multirow{2}{*}{ : } & & 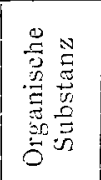 & $\begin{array}{l}: \frac{\Xi}{0} \\
0 \\
0 \\
\frac{2}{2} \\
0 \\
0\end{array}$ & 离 & 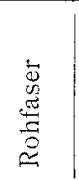 & 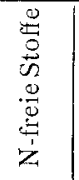 & 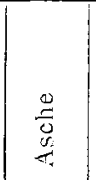 & 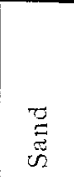 \\
\hline & & \multicolumn{7}{|c|}{$\mathrm{Gram} \mathrm{me}$} \\
\hline \multirow{4}{*}{ I } & Nahrung & $112 \cdot 72$ & $26 \cdot 22$ & $52 \cdot 12$ & \multicolumn{2}{|r|}{$\cdot 00$} & $2 \cdot 34$ & - \\
\hline & Koth. & $7 \cdot 50$ & $1 \cdot 68$ & 0.79 & \multicolumn{2}{|c|}{4.01} & $0 \cdot 91$ & $0 \cdot 11$ \\
\hline & Verdaut. & $105 \cdot 22 \mid$ & $24 \cdot 54$ & $51 \cdot 33$ & \multicolumn{2}{|c|}{$28 \cdot 03$} & $1 \cdot 43$ & - \\
\hline & Procente verdaut. & $93 \cdot 3$ & $93 \cdot 6$ & $98 \cdot 4$ & \multicolumn{2}{|c|}{$87 \cdot 4$} & $61 \cdot 1$ & - \\
\hline \multirow{4}{*}{ II } & Nahrung & $85 \cdot 88$ & $17 \cdot 92$ & $42 \cdot 14$ & \multicolumn{2}{|c|}{$0.03 \mid 24.07$} & 172 & - \\
\hline & Koth... & $6 \cdot 85$ & $1 \cdot 81$ & $0 \cdot 86$ & \multicolumn{2}{|c|}{$3 \cdot 01$} & $1 \cdot 09$ & 0.08 \\
\hline & Verdaut & $79 \cdot 03$ & $16 \cdot 11$ & $41 \cdot 28$ & \multirow{2}{*}{\multicolumn{2}{|c|}{$\begin{array}{l}20 \cdot 09 \\
83 \cdot 3\end{array}$}} & $0 \cdot 63$ & - \\
\hline & Procente verdaut... & $92 \cdot 00$ & $89 \cdot 9$ & $97 \cdot 9$ & & & $36 \cdot 6$ & - \\
\hline \multirow{4}{*}{ III } & Nahrung .. & $102 \cdot 56$ & $16 \cdot 43$ & $36 \cdot 84$ & \multicolumn{2}{|c|}{$\underbrace{0.03 \quad 47 \cdot 64}$} & $1 \cdot 62$ & - \\
\hline & Koth.... & $6 \cdot 30$ & $2 \cdot 87$ & $1 \cdot 05$ & \multirow{2}{*}{\multicolumn{2}{|c|}{$\begin{array}{r}1 \cdot 43 \\
46 \cdot 24\end{array}$}} & $0 \cdot 88$ & 0.07 \\
\hline & Verdaut . & $96 \cdot 20$ & $13 \cdot 57$ & $35 \cdot 79$ & & & 0.74 & - \\
\hline & Procente verdaut... & $93 \cdot 8$ & $82 \cdot 5$ & $97 \cdot 1$ & \multicolumn{2}{|c|}{$96 \cdot 5$} & $45 \cdot 7$ & - \\
\hline \multirow{4}{*}{ IV } & Nahrung & $97 \cdot 31$ & $16 \cdot 53$ & $37 \cdot 36$ & \multicolumn{2}{|c|}{$0.05 \mid 41 \cdot 87$} & $1 \cdot 50$ & - \\
\hline & Koth. & $5 \cdot 69$ & $2 \cdot 50$ & $1 \cdot 22$ & \multicolumn{2}{|c|}{$0 \cdot 95$} & 0.94 & $0 \cdot 08$ \\
\hline & Verdaut. & $91 \cdot 62$ & $14 \cdot 03$ & $36 \cdot 14$ & \multicolumn{2}{|c|}{$40 \cdot 97$} & 0.56 & $\ldots$ \\
\hline & Procente verdaut... & $94 \cdot 1$ & $84 \cdot 8$ & $97 \cdot 3$ & \multicolumn{2}{|c|}{$97 \cdot 7$} & $37 \cdot 3$ & - \\
\hline
\end{tabular}

Die Ergebnisse obiger Tabelle zeigen zunächst, dass die Verdaulichkeitsziffer der einzelnen Nährstoffe durchwegs eine hohe und für alle Perioden eine ziemlich gleichartige ist.

Die Anwesenheit von sehr geringen Sandmengen im Kothe, und zwar in allen Perioden, trotzdem das Futter überall sandfrei war, ist eine Erscheinung, die schon vielfach bei Fütterungsversuchen von anderen Forschern constatirt wurde.

Vermuthlich dürfte der Hund beim Herumlaufen im Laboratorium, insbesondere aber im Hofe mit seiner Schnauze den Boden berihrt haben, wodurch dann leicht bei der Gewohnheit 
des Hundes, seine Schnauze zu belecken, Sandtheilchen verschluckt sein konnten.

Bei unseren Berechnungen haben wir den Sandgehalt gar nicht in Betracht gezogen. Für uns ist besonders die Thatsache wichtig, dass der Verdauungscoëfficient von Rohfett ganz ausserordentlich hoch ist.

Die sehr geringe Differenz zwischen den Perioden III und IV, welche einen höheren Verdauungscoëfficienten für die Margarinperiode liefert, kann man ohne Weiteres vernachlässigen und annehmen, dass die Ausnützung des Fettes in beiden Periođen vollständig gleich war.

Bei den Perioden I und II ist eine geringe Differenz $z \mathrm{u}$ Gunsten der Naturbutter $z u$ bezeichnen, die aber ebenfalls in den Grenzen der Versuchsfehler liegt, so dass die erhaltenen Zahlen auch in diesen Perioden für eine gleiche Verdaulichkeit der beiden Fette sprechen.

Wir gelangen somit auf Grund unserer vergleichenden Fütterungsversuche zu dem Ergebnisse, dass unter gleichen Verhältnissen ganz reines Margarin dieselbe Verdaulichkeit und denselben Nährwerth wie ganz reine Naturbutter besitzt.

Als Gesammtresultat ergibt sich, dass, um zu verlässlichen Schlüssen über die Verdaulichkeit und den Nährwerth bei Naturbutter und Margarin zu gelangen, ausschliesslich reine Materialien verwendet werden müssen. Des Weiteren sollen die Perioden nicht kurz gewählt, sondern zum mindesten auf eine Dauer von acht Tagen ausgedehnt und die Versuche unter denselben Cautelen und den natürlichen Lebensverhältnissen des Versuchsthieres angepasst, durchgeführt werden.

Endlich dürfen die täglich zu verfütternden Fettmengen die Grenzen der Verdauungsfähigkeit des Versuchsobjectes nicht überschreiten.

Bei genauer Einhaltung aller dieser Bedingungen gelangt man zu dem Ergebnisse, dass das reine Margarin den gleichen Verdaulichkeitscoëfficienten und gleichen Nährwerth wie reine Naturbutter besitzt. 